\title{
A Framework for Integrated Optimization of Product Development Process
}

\author{
Min $\mathrm{Li}^{1}$, Yaluo Yang ${ }^{1}$, Jing $\mathrm{Bai}^{2}$ and Xiansheng Qin $^{2}$ \\ ${ }^{1}$ School of Mechatronics, KunMing University of Science and Technology, Kunming \\ 650093, P.R.China leah163@163.com yyl6xg@163.com \\ ${ }^{2}$ School of Mechatronics, Northwestern Polytechnical University, Xi'an 710072, P.R. China \\ bj0625@hotmail.com xsqin@mwpu.edu.cn
}

\begin{abstract}
The integrated optimization of product development process (PDP) is a critical strategy to improve the product development capability. It should be targeted for time, quality, cost, service and environment. Based on review of existing optimizing methods and tools applied in PDP, which are sorted in their optimizing goals, the problems and difficulties of PDP integrated optimization are put forward and analyzed in this paper. Then, we present and elaborate the fundamental principles for integrated optimization of PDP. According to these fundamental principles, at the end of this paper, a preliminary framework for PDP integrated optimization is established.
\end{abstract}

Keywords: Product life cycle systems, Life cycle process, Process-oriented system architectures, Multidisciplinary design optimization (MDO)

\section{INTRODUCTION}

Product development process (PDP) is a collection of activities which link the engineering techniques, methods, tools and people together and apply them into the product development practice. PDP involves technology and management issues [1]. How to improve the capability of product development, including technology level and management level, is very important in a long period in China $[2,3]$.

PDP, full of different functions and objects, constitutes a complicated big system. The elements influencing this multi-objective and multi-constraint system are changing dynamically and stochastically. Theories suitable for this kind of complicated system have not been developed till now. One prime tendency of PDP evolving mode will be the integrated optimization of PDP [2]. This paper presents the primary research results on multi-goal integrated optimization of PDP oriented in product life cycle, which focus on the managerial activities in PDP. 


\section{VARIATION OF PRODUCT DEVELOPMENT CIRCUMSTANCE}

The variation of enterprises' internal and external circumstances brought about the corresponding variation of product development circumstance. It includes:

- Higher requirements of product development: The changing world-wide market require more than ever the products to have shorter time-to-market $(T)$, higher quality $(\mathrm{Q})$, lower cost $(\mathrm{C})$, better service (S) and cleaner environment impact (E). Knowledge-based economy makes the product innovation, Knowledge $(\mathrm{K})$, a new hotspot of competition.

- Broader contents of product development: Developers had to extend their attention to involve the whole product life cycle, including better customer services and less negative impact on eco-environment. This adds to the PDP complexity.

- More scientific mode of product development: To eliminate the shortcomings and low efficiency caused by 'Throw Over the Wall' relationship in traditional PDP, Concurrent Engineering (CE), Integrated Product Team (IPT) and Integrated Product Development (IPD) are adopted to help the developers consider the later stages in product life cycle at the very beginning of PDP.

- More advanced techniques used in product development: Rapid Prototyping (RP), Virtual Manufacturing (VM), Computer aided techniques (CAX) and Design for $\mathrm{X}$ (DFX), etc. provide the stronger technical support for PDP. At the same time, the rapid growing of information technology, such as network and database technology, changed drastically the means of data exchange, store and access in PDP.

\section{EXISTING METHODS OF PDP OPTIMIZATION}

\subsection{Time-oriented Optimizing Techniques $[4,5]$}

The concept of CE was imposed originally as a method of shortening PDP time. $\mathrm{CE}$ increases greatly the product manufacturability and assemblability, thus decreases the time spent on rework. RP, a milestone of manufacturing technology, was developed in the past 20 years. It transforms rapidly the design into product prototype with certain structure and functions, or directly product parts. RP enables the product design to be evaluated, tested and improved quickly, then completes the PDP to satisfy the market.

\subsection{Quality-oriented Optimizing Techniques [6-8]}

For improving the product quality, quality control methods and tools employed in PDP includes: 
- Design quality control: Robust Design (RD), Failure Mode and Effect Analysis (FMEA), Design of Experiments (DOE), DFX, etc.

- Manufacturing quality control: Statistical Process Control (SPC), Quality Data Acquisition (QDA), etc.

- Whole process quality control: Quality Function Deployment (QFD), Total Quality Management (TQM), etc.

Contemporary product quality means to meet all kinds of costumer needs in the whole product life cycle. Up to now, researches on quality-oriented optimizing tools consider mostly design quality and manufacturing quality. Enough attention should be paid to the development of methods and tools optimizing the use and reuse of products, resources consumption, and impact on eco-environment during the manufacturing stage.

\subsection{Cost-oriented Optimizing Techniques}

The application of Advanced Manufacturing Technology (AMT) decreased greatly the time needed to develop product, but it has nothing to do with optimizing product development cost. Numerous researches were carried out to estimate and cut down the cost. German scholars analyzed cost structure and classification, compared and evaluated different design schemes by relative cost, translated design features into cost features using design methodology and cost-controlling design idea, and proposed customer-oriented target cost accounting method. American scholars suggested cost model based on the regression statistical results of product data. Besides, lots of scholars in Japan, Swiss and UK devoted themselves to manufacturing cost estimation. Chinese scholars have also done much work in this field, but with the limitation of poor data reliability, shortage of research funds and manpower, and immensity of workload and difficulty, the progress is slow, and only theoretical analysis and computation can be done to belp design for cutting down the cost. The analytical results are mostly qualitative, and the few quantitative analyzes are statistical. The idea of design for cost can not be incorporated into an integrated $\mathrm{CAD}$ system running through the whole design process. Therefore, a cost information model supporting the whole design process, especially the scheme design and general structure design, becomes the bottleneck of design for low cost.

The development of life cycle engineering extends the product cost components to the whole life cycle. The methods used to compute the life cycle cost (LCC) include Design for Cost (DFC) and Activity-Based Costing (ABC).

\subsection{Service-oriented Optimizing Techniques}

Being the core strategy of today's enterprises, product service has becoming one of the important content in the competition. Customer service, like timely technical support, customer communication, and reliable usage promise, has been more and more important. Enterprises need to win the customer and then the market through the improvement of service quality and new service pattern. 
Traditional customer service optimization is implemented primarily by enhancing the management of service stuff and service system, such as post training, service attitude improvement and service network expansion. But enterprises need more than management measures to stand in the competitive market. The development of computer and network technology brought a good chance for optimizing service.

Customer Relationship Management (CRM) is a new management mechanism to polish up the relationship between enterprises and customer. Through timely and various communication and exchange between enterprises and customer, long-term and benign relation is established. This relation that means more beyond merely deal helps keep the valuable customer and attract more potential customer. CRM is a set of human-computer interactive system as well as a solution aiming at the harmonization of product marketing, sales, service and support. It includes general CRM and Electronic Commerce supported CRM (eCRM). The CRM software has been applied to many industrial fields like manufacturing, finance, insurance, software, etc.

\subsection{Environment-oriented Optimizing Techniques [9]}

The world-wide attention to environment pollution needs enterprises to adopt clean manufacturing $(\mathrm{CM})$ technology, green materials and energy sources to replace traditional manufacturing mode whose negative environmental impact can not be ignored. The purpose of clean manufacturing is to utilize the resources to the most efficiency and produce castoff to the least amount. Green design is a critical component in the clean manufacturing technology. It lists characters of the resources consumption decrease, easy to disassembly and recycle, eco-environment-friendly as the same design goals as product performance, quality, life-span and cost, and the product design should be completed in the production. With the support of Chinese National Science \& Technology Committee and National Natural Science Foundation of China, some university and institute began the research items on clean manufacturing technology, which include: 'research on environmental green technology evaluate system', 'research on recyclable design theory and key techniques of mechanical product and the recycle indices evaluate system', 'research on the system structure of sustainable CIMS (S-CIMS), system structure and application strategy of clean production system, and clean production information management system', etc.

\section{THE DIFFICULTIES OF PDP INTEGRATED OPTIMIZATION}

The pursuing of TQCSEK will never end. Although the PDP optimizing theories and techniques has made remarkable progress after long period development, there are still many difficulties which block their application.

\subsection{Absence of Systematic and Integrative Research on PDP Optimization}




\section{Existing techniques only optimize part of PDP}

From the earliest application of numerical optimization methods in engineering design, to presently the disperse stochastic variable optimization, structure optimum design, intelligent optimization, optimum modeling and optimum methods in complicated systems, optimization techniques are mostly employed in the design stage of PDP. At the same time, for other stages in PDP, the optimization is independent; focus on only one or two of them. For example, DFX optimizes the product design oriented in the different later stages, VM and $\mathrm{CM}$ optimizes the product manufacturing. While BPR and CE take the product life cycle into account, they optimize only the PDP organizing and management. Researches on optimizing the whole PDP are only limited in fundamental theories, methods and models. To our best knowledge, there is not an effective analyzing and controlling optimizing tool which could complete the entire PDP optimization, from the PDP model establishment, management, to PDP optimum results presentation and validation.

2. Existing techniques only optimize for single objective

There exists contradiction and competition among the objectives of PDP integrated optimization, so it's almost impossible to reach the optimum points at the same time. Enterprises have to weigh the multi objectives according to their circumstances and certain products, and then make the most suitable decision. Therefore, an optimizing tool permits the integration and tradeoff among multi objectives is indeed what the enterprises cry for. Existing methods optimize only one or two of the multi objectives, for example, LCC and ABC optimize the PDP cost, QFD and SPC optimize quality, and CM optimize the environment impact. These single-objective optimizing methods can not evaluate a PDP decision from every facet, so fail to provide comprehensive information to help enterprises make favorable choices.

\subsection{Absence of Integrated Evaluation Indices System}

Researches on enterprises' evaluation indices systems have been done for a long time, and different methods and indices are employed to appraise the enterprises' performances. Relative values, like productivity and investment yield rate, has gradually replaced absolute values, like production value and profit. For a long period, financial indices have been the chief content in the enterprises' performances appraisal systems. Recently, non-financial indices are introduced into the systems, such as Balanced Score board proposed by American scholars Robert Kaplan and David Norton which use four indices, financial, customer, internal business processes and learning and growth, to appraise an organization's performance.

Evaluation indices now used to evaluate an enterprise's business processes are mostly statistical date processing after the processes are finished. Without decomposing, the indices can not be allocated to activities, stuff, and resources on low layer in the PDP hierarchical structure, so can not evaluate all the sub-process performances systematically and objectively. In a complex system, components play different roles and have different impacts on the system performance. These impacts may be indirect and they are transferred from bottom to the top layer. To a complex big system like PDP, indices must be decomposed to appraise the system performance 
scientifically. This hierarchical indices system has not been found in the open literature till now.

\subsection{Absence of Dynamic Control and Optimization Tools for PDP}

In knowledge-based economy, rapidly changing market and enterprises' circumstance require fast response and adjustment. Traditionally, business processes appraisal methods are executed after the processes are completed. Thus, the appraisal results may not be appropriate to the future processes. Because of the custom-tailor, little batch and diversification product demand, as well as the shorter PDP time, what the enterprises really want is breaking down the general goals into the sub-processes goals to guide the execution of them, analyzing the impact of unexpected situation at the real time, then taking corresponding measures to adjust the running process and guide the later processes, so as to avoid the loss to a maximum extent and optimize the process performance. It is necessary and urgent to develop such an optimizing tool to help real-time, dynamic adjustment of sub-processes in PDP.

\section{BASIC PRINCIPLES OF PDP INTEGRATED OPTIMIZATION}

There are numerous external or internal factors influencing the complex PDP system. These dynamically changing factors link together and depend on each other, so integrated evaluation and optimization are needed to reflect the PDP performance objectively and accurately. The main idea of PDP integrated optimization is to regard PDP as an integer and integrative process, manage and control it entirely and comprehensively, thus to suggest and analyze the integrative optimum solution of PDP.

Design of process parameters in PDP determines the whole performance of PDP and then relates the success of product development. A parameter optimum to a single process may not be optimum to the whole PDP. A parameter optimum to a single performance index may not be optimum to TQCSEK. So design of process parameters should stand in full view and consider integratedly the whole performance of PDP. Enterprises cry for a tool to design the process parameters which are optimum to six performance indexes or overall performance of PDP. At a different angle, TQCSEK indexes are deployed into PDP hierarchy to acquire the parameter values in various levels.

On the other hand, there should be some technical key points in PDP, namely key sub-processes and key parameters of them, which determine the performance of PDP. The PDP performance could not be assured if these key points do not work well. Besides, there should be some chance points, certain sub-processes and key parameters of them, for PDP performance optimization, whose improvement could improve the PDP performance remarkably. If these technical key points and chance points could be confirmed before the execution of PDP, PDP performance will surely be greatly advances by emphasizing the quality assurance and control of the key 
points as well as adjusting the chance points carefully, and then PDP performance is optimized integratedly.

\subsection{Break Down Level by Level}

Breaking down level by level is proved one of the most effective methods as researching complex systems. As a kind of complex process system, there exists clear hierarchy within PDP. This hierarchy exists in two ways: Product development is organized on corresponding administrative level and the task of PDP is broken into sub-tasks executed on various level. For example, as viewed from organization, PDP may be broken into process on business level, company level, project level, team level and individual level; Design of product may be broken down into design of components and parts, and technology design may be broken into technology flow design and procedure design. Accordingly, management of PDP is hierarchical. The number of levels is decided by the complexity of product and product developing, with a view of the requirement of company overall harmonization. Content and objects are various on various levels, so management and optimization of PDP should be carried out on various levels.

\subsection{Process Based}

In the final analysis, product development target is realized through the execution of every process. With a view of process, PDP is made up of a series of processes whose perplexing relation shapes a network structure. This process network structure is hierarchy and its execution characters concurrent macroscopically and serial microscopically. Each process has its specific executing parameters or indexes, such as task, time and resources needed, no matter the level it locates. When resources and activities are managed as process, the inputs, outputs and actions could be identified and confirmed, and quality of each item be measured and controlled; At the same time, relations, reactions and interfaces with other processes are confirmed for the harmonization and compatibleness of whole organization. Therefore, process based method provides conditions for thorough and effective control of PDP, and a way to eliminate the ramparts among functional departments and administrative levels, by which transfer of process information is accelerated and enterprises could react more quickly to dynamic changes in PDP.

\subsection{Process Programming}

For PDP integrated optimization, transfer relationship among performance indexes of various sub-processes should be established to deploy the six indexes into PDP hierarchy level by level. This could:

- identify the process key characteristics to form PDP evaluation index system which acts as control points before process execution and analysis or check points when process is executing or after process finishes; 
- quantify indexes of process key characteristics to confirm the parameters and more important, to look for the chance points and the optimum parameters for PDP integrated optimization.

Goals above attract our attention to Quality Function Deployment (QFD) which employs many tools to structure the inter-relations for decision-making. QFD is not only a kind of quality tool, but also a programming method. It establishes quality transfer relation among various sub-processes in PDP through product quality deployment. This idea suggests us to establish performance indexes transfer relation among various sub-processes through PDP performance indexes deployment (Figure 1).

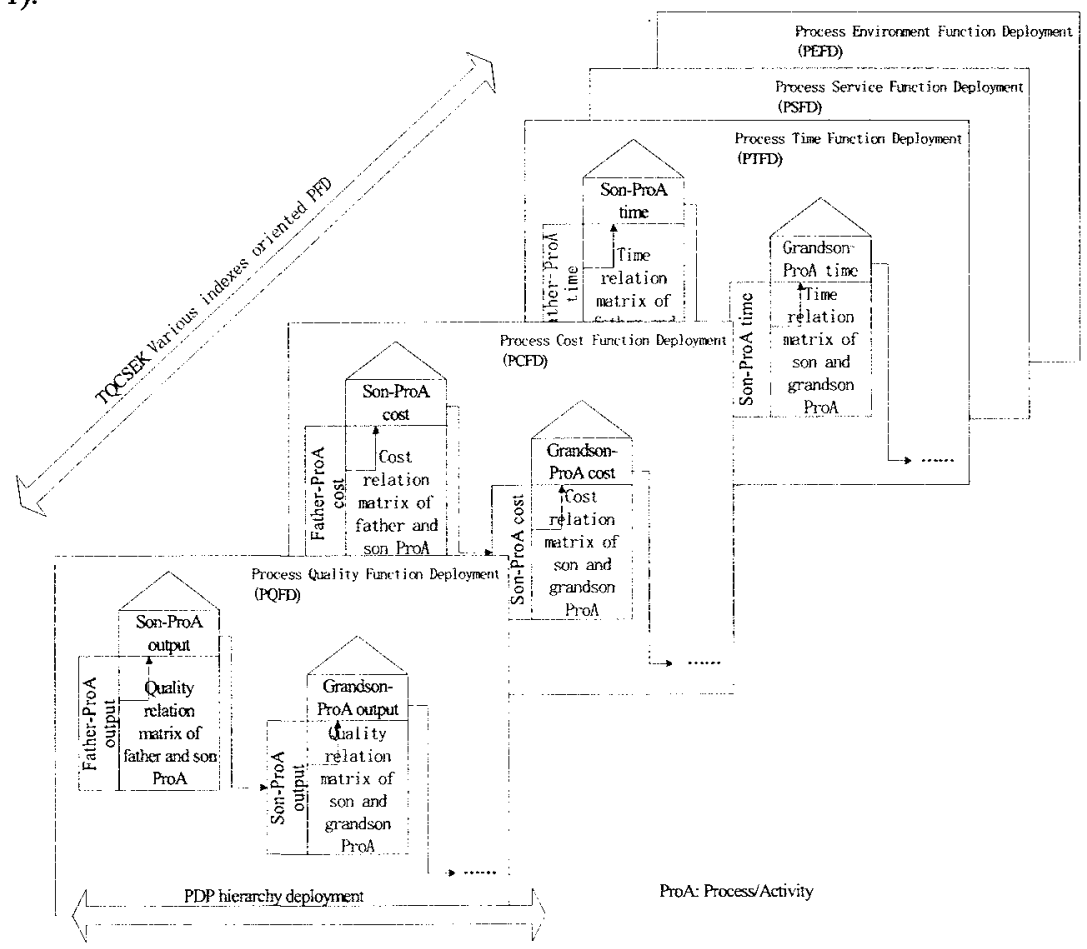

Figure 1. PDP Deployment Hierarchy Model

Considering integratedly every level in PDP hierarchy model, a solid threedimensional model is formed and we call it solid integrated optimization house(Figure 2). 


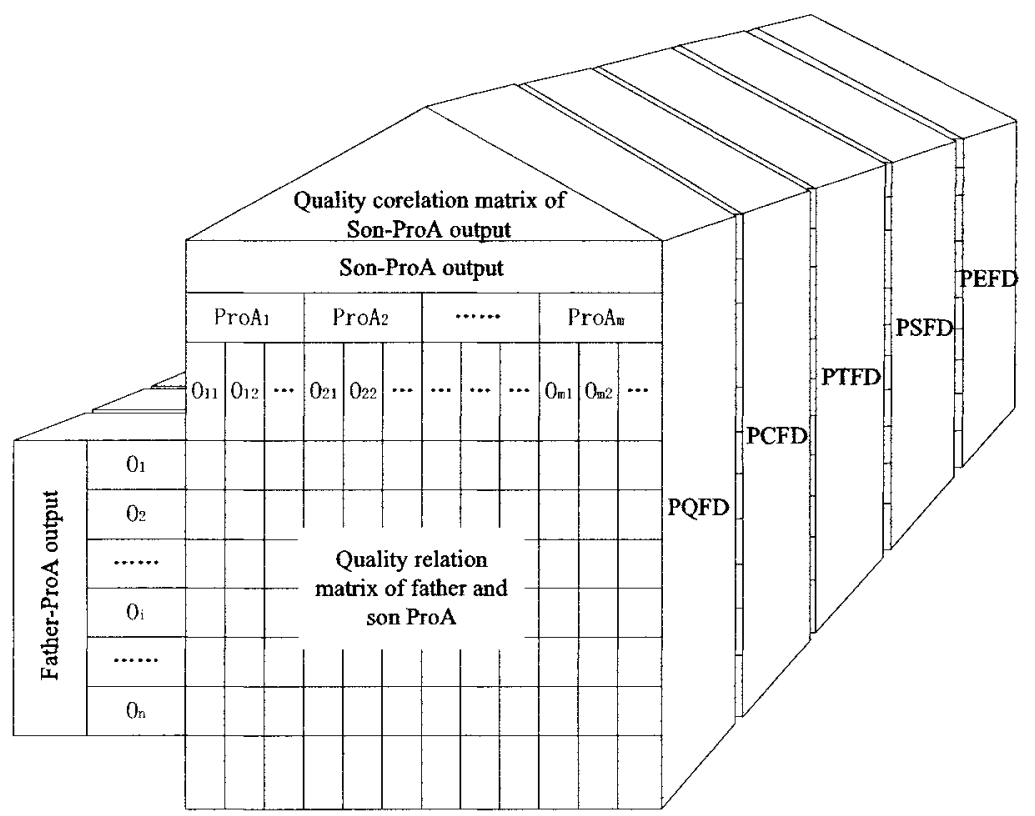

Figure 2. PDP Solid Integrated Optimization House

\section{CONCLUSIONS}

PDP is a multi-level complex system characterized by collaboration, dynamic, and gradualness. Single-objective or local optimization is far from meeting the application requirement. At the early stage of PDP, systematically taking the performance indexes like time, cost and quality etc. into account, programming and deploying process key characteristics and parameters, and then consciously precontrolling and checking these key points would highly improve PDP performance. The process based, PDP hierarchy programming principle proposed in this paper, is only a little pathbreaking work for PDP integrated optimization.

\section{REFERENCES}

1. G. Xiong, Theory and Practice of Current Engineering (Tsinghua University Press: Beijing, 2000).

2. C. Wu, Introduction to Contemporary Integrated Manufacturing System (Tsinghua University Press: Beijing, 2002).

3. G. He, Machinery Manufacturing Industry of China at the Turn of the Centuries, China Mechanical Engineering. Volume 9, Number 1, pp.2-9, (1998). 
4. P.S. Adler and A. Mandelbaum, From Product to Process Management: an Empirically-based Framework for Analyzing Product Development Time, Management Science. Volume 41, pp.458-484, (1995).

5. G. Burchill and C. Fine, Time Versus Market Orientation in Product Concept Development: Empirically-based Theory Generation, Management Science. Volume 43, pp.465-478, (1997).

6. S.A. Bisgaard, Conceptual Framework for the Use of Quality Concepts and Statistical Methods in Product Design, Journal of Engineering Design. Volume 3, Number 1, pp.31-47, (1992).

7. D.P. Clausing, Total Quality Development (John Wiley \& Sons, Inc: New York, 1994)

8. P. Das, Concurrent Optimization of Multiresponse Product Performance, Quality Engineering. Volume 11, Number 3, pp.365-368, (1999).

9. J. Cramer, Early Warning: Integrated Eco-efficiency Aspects into the Product Development Process, Environmental Quality Management. Volume 10, Number 2, pp.1-10, (2000). 